\title{
AVALIAÇÃO DA AUTORREDUÇÃO DE MISTURAS DE PÓ DE ACIARIA ELÉTRICA E COQUE DE PETRÓLEO POR PERDA DE MASSA E DIFRAÇÃO DE RAIOS $X^{*}$
}

\author{
Anderson Azevedo Agra ${ }^{1}$ \\ Felipe Buboltz Ferreira² \\ Bruno Deves Flores ${ }^{3}$ \\ Antônio Cezar Faria Vilela ${ }^{4}$
}

\section{Resumo}

O Pó de Aciaria Elétrica (PAE) é um resíduo sólido gerado durante a fabricação de aços em usinas com Forno Elétrico a Arco (FEA). Esse resíduo contem quantidades apreciáveis de metais, tais como ferro e zinco, tornando sua reciclagem interessante não só do ponto de vista ambiental como econômico. Assim, esse trabalho teve como objetivo avaliar a autorredução de misturas de pó de aciaria elétrica e coque verde de petróleo (CVP). Para isso, misturas e pelotas de PAE:CVP, com variados teores de redutor, foram submetidas a ensaios de autorredução em termobalança e, na forma de pelotas autorredutoras, numa retorta aquecida em forno elétrico vertical. A redução das amostras foi analisada a partir da perda de massa das misturas. Além disso, a redução das pelotas autorredutoras foi observada através de análises de difração de raios $X$. Nestas análises foi possível verificar decomposição da Franklinita e a redução dos óxidos de Ferro e Zinco.

Palavras-chave:Autorredução; PAE; Difração de raios x; Termobalança.

\section{EVALUATION OF SELF-REDUCTION OF ELECTRIC ARC FURNACE DUST AND PETROLEUM COKE MIXTURES BY WEIGHT LOSS AND X RAY DIFFRACTION}

\begin{abstract}
The Electric Arc Furnace Dust (EAFD) is a solid waste generated in steelmaking by plants with Electric Arc Furnace (EAF). This waste contain quantities of valuable metals, such as iron and zinc, making its recycling interesting not only under an environmental concern but in an economic way too. Thus, this work aims to evaluate the self-reduction of EAFD and petroleum coke (CVP) mixtures. Thereby mixtures and pellets, composed of EAFD and CVP, with different reductant contents were submitted to heating tests in thermobalance and laboratory furnace. The reduction of the samples was carried out by the mixtures' weight losses. Furthermore, reduction of the self-reducing pellets was analysed by $X$ Ray Diffraction. In these analysis it was possible to verify franklinite decomposition and reduction of iron and zinc oxides.

Keywords: Self-Reduction; EAF Dust; X Ray Difraction; Thermobalance.

\footnotetext{
Graduando de Eng. Metalúrgica, LaSid/DAMET/EE/UFRGS, Porto Alegre, RS/Brasil.
} Eng. Materiais, Mestrando, LaSid/PPGE3M/EE/UFRGS, Porto Alegre, RS/Brasil.

Eng. Metalurgico, Doutorando, LaSid/PPGE3M/EE/UFRGS, Porto Alegre, RS/Brasil.

Prof. Dr-Ing., LaSid/DAMET/PPGE3M/EE/UFRGS, Porto Alegre, RS/Brasil.
\end{abstract}




\section{INTRODUÇÃO}

O Pó de Aciaria Elétrica (PAE) é um resíduo sólido gerado durante a fabricação de aços em usinas que utilizam Forno Elétrico a arco (FEA). A geração deste resíduo representa cerca de 1 a $2 \%$ de toda a carga carregada no FEA [1-3]. Dados da "Worldsteel" apontam que de 2005 a 2014 a produção de aço via FEA aumentou em $12 \%$ no Brasil e em 15\% no mundo [4]. Esta alta geração de resíduo aliada a crescente utilização do FEA traz o problema de acúmulo deste material. Atualmente metade de todo o PAE do mundo é destinado a aterros industriais [5]. A destinação para aterros é explicada por dois fatores: primeiramente pelas características do $\mathrm{PAE}$, que por conter teores de cádmio e chumbo elevados o tornam um resíduo classe I - perigoso (norma NBR 10004). O outro fator é devido à inexistência de uma aplicação ou processo no qual esteja completamente estabelecido à reutilização ou reciclagem do PAE. Esta destinação a aterros acarreta problemas tanto no contexto ambiental, podendo trazer um passivo ambiental, quanto no contexto econômico por gerar custos.

Apesar de sua composição poder ser alterada significantemente dependendo o tipo de aço fabricado pela a usina que lhe deu origem, em diversos estudos foram demostrados que o PAE é constituído basicamente de óxidos metálicos, majoritariamente óxidos de ferro e zinco [6;7]. Além da presença em menores quantidades de óxidos de outros elementos, tais como chumbo, cadmio, estanho e etc. A presença de óxidos de interesse metalúrgico, como ferro e zinco, na composição do PAE, aliado a busca de solucionar o passivo ambiental que a destinação a aterros gera, é o que o torna um atrativo para reciclagem.

Chang (1999) relata a existência de um grande número de tecnologias desenvolvidas e aplicadas para a reciclagem do PAE, sendo elas pirometalurgicas, hidrometalurgicas e de vitrificação. Os processos pirometalurgicos são os mais empregados atualmente. Nesses a recuperação dos metais contidos no PAE se dá através da redução carbotérmica dos óxidos do material. Dentre os processos destaca-se o processo Waelz, principal método de reciclagem de pó de aciaria elétrica. Nesse processo pelotas autorredutoras contendo PAE, são carregadas em um forno rotativo visando principalmente à geração de concentrados de zinco.

Apesar da existência de soluções tecnológicas para a reciclagem de PAE em usinas siderúrgicas integradas, a reciclagem se torna um desafio quando estas não possuem unidades de redução, ou que não tem a possibilidade de destinar o seu resíduo para plantas específicas de recuperação de PAE [9]. Buzin (2009) e Bagatini (2011) estudaram a possibilidade de se recuperar resíduos ferrosos através do uso de briquetes autorredutores diretamente em fornos elétricos a arco. Esses autores sugerem que tal abordagem surge como uma alternativa para caso onde não haja unidades de redução. Assim a introdução de aglomerados autorredutores pode ser uma possibilidade interessante para a recuperação de PAE [10;11].

A produção aglomerados autorredutores busca, basicamente, fazer uma mistura íntima dos óxidos metálicos e um agente redutor de modo que, quando sob aquecimento em elevadas temperaturas, a alta pressão parcial dos reagentes leve a altas velocidades de reação [11]. Estas altas velocidades de reação permitem a estes aglomerados ter um tempo de residência muito curto no forno. Diversos processos utilizam a tecnologia de autorredução. Estes processos podem ser em fornos de soleira rotativa, comumente chamados de RHF (Rotary Hearth Furnace), tais como, RedSmelt, ITmk3, Inmetco, FastMet, IDI, Maumee ou em fornos de cuba tais como o Tecnored e o Oxicup. 
Este trabalho tem como objetivo avaliar a autorredução de pelotas constituídas de pó de aciaria elétrica e coque de petróleo (CVP), por perda de massa e análise qualitativa das fases via difração de raios- $x$, para diferentes teores de agente redutor.

\section{MATERIAIS E MÉTODOS}

\subsection{Matérias-primas utilizadas nos ensaios de autorredução}

\section{Pó de aciaria elétrica}

O pó de aciaria elétrica oriundo de uma usina semi-integradada do Rio Grande do Sul foi amostrado e caracterizado em trabalho anterior [7]. O pó utilizado no estudo é composto majoritariamente por zinco e ferro (Tabela 1). Esses elementos encontram-se majoritariamente distribuídos nas fases Franklinita $\left(\mathrm{ZnFe}_{2} \mathrm{O}_{4}\right)$, Zincita $(\mathrm{ZnO})$ e Magnetita $\left(\mathrm{Fe}_{3} \mathrm{O}_{4}\right)$ (Tabela 2).

Tabela 1. Composição elementar do pó de aciaria.

\begin{tabular}{ccccccccc}
\hline & $\mathrm{Zn}$ & $\mathrm{Fe}$ & $\mathrm{Mn}$ & $\mathrm{Ca}$ & $\mathrm{Mg}$ & $\mathrm{Pb}$ & $\mathrm{Na}$ & $\mathrm{C}$ \\
Composição & 34,23 & 22,80 & 2,75 & 2,49 & 1,76 & 1,10 & 1,00 & 0,91 \\
\cline { 2 - 9 } elementar & $\mathrm{K}$ & $\mathrm{Al}$ & $\mathrm{Cu}$ & $\mathrm{Ba}$ & $\mathrm{Cd}$ & $\mathrm{Ni}$ & $\mathrm{Sn}$ & \\
(\% em massa) & 0,97 & 0,20 & 0,15 & 0,05 & 0,03 & 0,01 & 0,01 & \\
\hline
\end{tabular}

Tabela 2. Principais fases oxidas do pó de aciaria.

\begin{tabular}{lcccc}
\hline (\% em massa) & ZnFe2O4 & $\mathrm{ZnO}$ & $\mathrm{Fe} 3 \mathrm{O} 4$ & Outras fases \\
\cline { 2 - 5 } & 36,9 & 30,2 & 7,9 & 25,1 \\
\hline
\end{tabular}

\section{Redutor}

Coque verde de petróleo (CVP) foi selecionado como redutor para os testes de autorredução. A Tabela 3 apresenta a análise imediata do redutor.

Tabela 3. Análise imediata CVP.

\begin{tabular}{cccc}
\hline \multirow{2}{*}{ Base seca (\% massa) } & Matéria Volátil & Cinzas & Carbono Fixo \\
\cline { 2 - 4 } & 10,1 & 0,6 & 89,3 \\
\hline
\end{tabular}

\subsection{Ensaios de autorredução de misturas PAE/CVP em termobalança}

Foram realizados, no Laboratório de Siderurgia - Lasid/UFRGS, ensaios em termobalança Netzsh, modelo 409 PC Luxx. As misturas, com massa de $250 \mathrm{mg}$, foram introduzidas em cadinho de alumina tipo copo $(37 \times 54 \mathrm{~mm})$, onde foram ligeiramente compactadas de forma manual. As amostras foram aquecidas a uma taxa de $30^{\circ} \mathrm{C} / \mathrm{min}$ até a temperatura de $600^{\circ} \mathrm{C}$, e mantidas nesta temperatura por 20 min. Essa primeira etapa isotérmica foi realizada com o intuito de eliminar das amostras a umidade e grande parte da matéria volátil do agente redutor. Após essa etapa de secagem e desvolatilização, as amostras foram aquecidas a uma taxa de $30^{\circ} \mathrm{C} / \mathrm{min}$ até $1100^{\circ} \mathrm{C}$ e mantidas nesta temperatura por $20 \mathrm{~min}$. Um fluxo de 100 $\mathrm{mL} / \mathrm{min}$ de nitrogênio foi introduzido no forno ao longo de todo o ensaio.

As misturas autorredutoras utilizadas no teste de termobalança foram produzidas com as seguintes relações PAE:CVP - 95:5, 90:10 e 85:15 (identificadas no trabalho COMO MPAE:CVP). 


\subsection{Pelotização das misturas autorredutoras de PAE/CVP}

Foram confeccionadas pelotas de $10 \mathrm{~mm}$ de diâmetro, manualmente, utilizando mistura de PAE:CVP com mesma relação mássica que a testada em termobalança. No entanto, de forma a conferir resistência mecânica às pelotas adicionou-se a essas misturas $5 \%$ em massa de cimento Portland(CIM). Este teor de cimento foi escolhido com referência a resultados obtidos em trabalho anterior [9]. A Tabela 4 mostra os percentuais mássicos de cada um dos constituintes das pelotas produzidas.

Tabela 4. Composição das pelotas autorredutoras de PAE/CVP (\% mássica).

\begin{tabular}{lccc}
\hline & PAE & CVP & CIM \\
$\mathrm{P}_{95 / 5}$ & 90,25 & 4,75 & 5 \\
$\mathrm{P}_{90 / 10}$ & 85,5 & 9,5 & 5 \\
$\mathrm{P}_{85 / 15}$ & 80,75 & 14,25 & 5 \\
\hline
\end{tabular}

A granulometria destes materiais foi analisada e visto que $75 \%$ do pó esta numa granulometria inferior a $2 \mu \mathrm{m}$. O CVP após adequação granulométrica apresentou-se com $65 \%$ do material com granulometria inferior a $150 \mu \mathrm{m}$. Já o cimento tem $80 \%$ do material menor que $11 \mu \mathrm{m}$.

\subsection{Ensaios de autorredução das pelotas}

Os testes de autorredução das pelotas produzidas (previamente secas em $110^{\circ} \mathrm{C}$ por 1h) foram realizados a partir da inserção de $40 \mathrm{~g}$ de amostra (12 \pm 1 pelotas) em retorta metálica, conforme esquematizado na Figura 1 (retorta de autorredução). Os testes de autorredução foram realizados a partir da inserção da retorta em forno elétrico vertical pré-aquecido. Durante os ensaios a temperatura das pelotas foi monitorada através de um termopar central e atmosfera no interior da retorta de autorredução foi mantida inerte a partir da injeção de $10 \mathrm{~L} / \mathrm{min}$ de nitrogênio. Os ensaios foram conduzidos mantendo-se a temperatura do forno constante em $1000^{\circ} \mathrm{C}$. As pelotas foram mantidas nessas condições durante períodos de $15,30 \mathrm{e}$ 60 minutos.

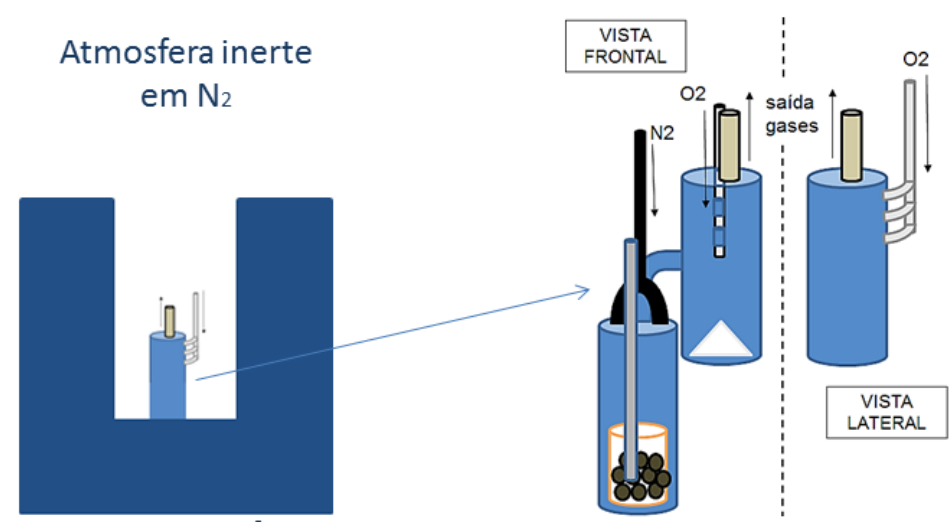

Figura 1. Aparato experimental para ensaios de autorredução das pelotas.

Após resfriamento das pelotas em atmosfera inerte, a perda de massa das amostras foi determinada. Além disso, as amostras submetidas a 60 minutos de ensaio foram analisadas por difração de raios $\mathrm{x}$. 


\section{RESULTADOS E DISCUSSÃO}

\subsection{Avaliação teórica da máxima perda de massa das misturas PAE:CVP}

Teoricamente, ao se submeter misturas PAE:CVP a temperaturas elevadas, a redução dos óxidos metálicos deve ocorrer via intermediários gasosos. Assim, a massa dessas misturas deve diminuir ao passo que os óxidos são reduzidos e o carbono, presente no redutor, é gaseificado. Para se avaliar a máxima perda de massa que uma mistura autorredutora pode ter é necessário determinar o teor de oxigênio ligado às fases passiveis de redução carbotérmica nas condições experimentais (oxigênio redutível), bem como o carbono necessário para reduzi-lo. $O$ valor de oxigênio redutível pode ser estimado partir da das fases oxidas presentes no PAE (dado pela Tabela 3), conforme discutido por Buboltz et al. [7]. Em resumo, o valor de oxigênio redutível do pó de aciaria é dado pela Equação (1).

$$
\text { Oredutível (Or) }=\% \mathrm{ZnFe} 204 \cdot \frac{4 \cdot \mathrm{MO}}{\mathrm{MZnFe} 2 \mathrm{O} 4}+\% \mathrm{ZnO} \cdot \frac{\mathrm{MO}}{\mathrm{MZnO}}+\% \mathrm{Fe} 304 \cdot \frac{4 . \mathrm{MO}}{\mathrm{MFe} 304}
$$

Onde Oredutivel corresponde ao percentual mássico de oxigênio ligado às espécies químicas $\mathrm{ZnFe}_{2} \mathrm{O}_{4}, \mathrm{ZnO}$ e $\mathrm{Fe}_{3} \mathrm{O}_{4}$ e $\mathrm{M}$ é a massa molar do oxigênio ou de cada um dos compostos $\left(\mathrm{ZnFe}_{2} \mathrm{O}_{4}, \mathrm{ZnO}\right.$ e $\left.\mathrm{Fe}_{3} \mathrm{O}_{4}\right)$. Para o PAE analisado este valor de oxigênio redutível é $17,9 \%$.

Admitindo-se que a redução dos óxidos metálicos ocorra de acordo com a reação estequiométrica (2), ou seja, que $1 \mathrm{~mol}$ de carbono seja necessário para se remover $1 \mathrm{~mol}$ de oxigênio redutível $(\mathrm{C} / \mathrm{O}$ em massa $=0,75)$, pode-se estimar a máxima perda de massa para cada uma das misturas. Essa perda máxima teórica pode ser calculando conforme a equação (3).

$$
\begin{gathered}
\text { MO }(\mathbf{s})+\mathbf{C}(\mathbf{s})=\mathbf{M}(\mathbf{s})+\mathbf{C O}(\mathbf{g}) \\
\% \text { Perda de Massa }=\frac{\left.\% \text { PAE na mistura.(\%0r } \% 0 \mathbf{r} \cdot \frac{\mathbf{C}}{0}+\% \mathrm{Zn}\right)}{100}
\end{gathered}
$$

$\mathrm{Na}$ equação (3) além da perda de oxigênio redutível e da massa de carbono associado a este que deixa o sistema como $\mathrm{CO}(\mathrm{g})$, conforme (2), também é contabilizado o desprendimento de Zinco. Isto porque após o zinco ser reduzido a zinco metálico ele não permanece no sistema, pois é imediatamente volatilizado $\left(907^{\circ} \mathrm{C}\right)$.

Essa estimativa é apresentada para as misturas autorredutoras utilizadas em termobalança e para os lotes de pelotas confecionados (tabela 6).

Tabela 6. Perdas de massa máxima teórica misturas autorredutoras e pelotas.

\begin{tabular}{cccc}
\hline & 5\% CVP & 10\% CVP & 15\%CVP \\
\hline Misturas (\%) & 54,9 & 56,7 & 58,5 \\
Pelotas (\%) & 52,1 & 53,8 & 55,6 \\
\hline
\end{tabular}

Nesta estimativa não é considerada a perda de massa devido ao teor residual da secagem de água utilizada na pelotização, umidade das matérias-primas, matéria volátil do redutor e redução de outros óxidos metálicos de teores menores, como o chumbo por exemplo. Além disto, é considerado que o cimento utilizado como ligante não reage durante o ensaio e tem sua massa mantida após os testes. Estes 
fatores tem uma contribuição menor para a perda de massa das misturas, frente aos considerados no cálculo teórico. Entretanto devido a estes fatores pequenas variações da previsão devem ocorrer.

\subsection{Testes de Autorredução em Termobalança}

Na figura 2 são apresentados os testes termogravimétricos para as misturas de PAE e diferentes teores de CVP, onde as linhas pontilhas referem-se aos valores de perda de massa das diferentes amostras estudadas e a linha sólida ao perfil térmico do ensaio.

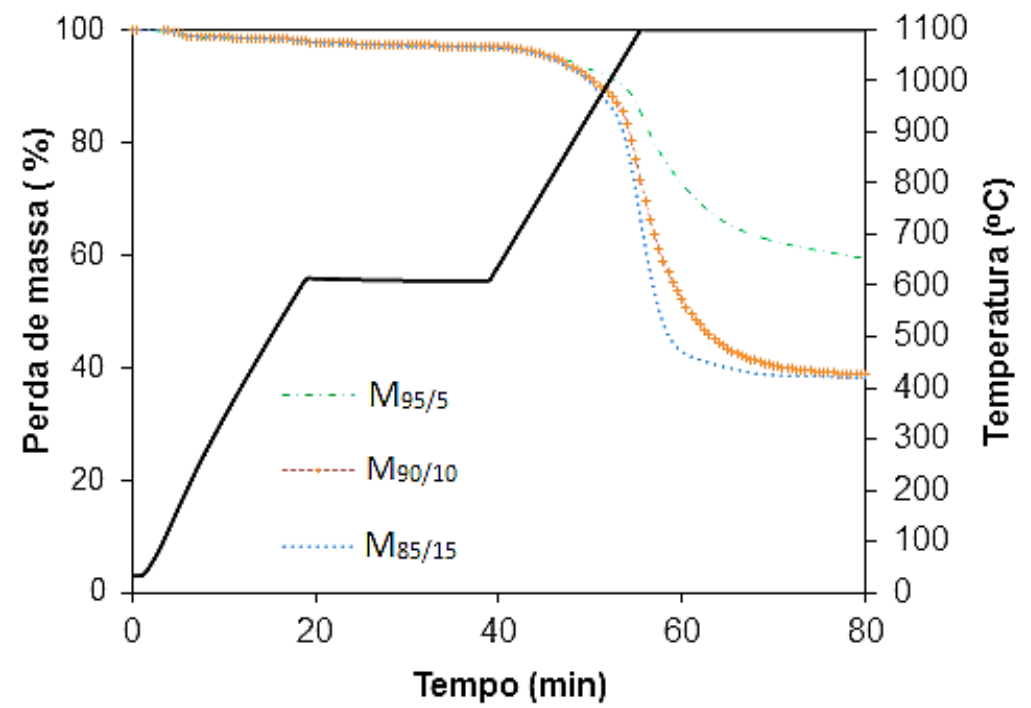

Figura 2. Testes de autorredução em termobalança. Onde a linha cheia representa o perfil térmico do ensaios e MPAE/CVP da a composição das misturas.

Observa-se que até atingir o fim da primeira etapa isotérmica $\left(600^{\circ} \mathrm{C}\right)$ todas as composições de misturas estudadas têm perdas de massa muito semelhantes, na ordem de 3\%. Esta perda de massa inicial ocorre devido liberação da umidade dos materiais e à desvolatização parcial do CVP. Apesar das composições das misturas serem diferentes, tanto a pequena quantidade de matéria volátil no coque de petróleo, quanto os pequenos teores do mesmo nas misturas dificulta verificar uma diferença significativa entre as diferentes misturas nesta parte inicial.

A perda de massa resultante do aquecimento das amostras no intervalo de temperatura entre 600 e $900^{\circ} \mathrm{C}$ é pequena e torna-se significativa apenas acima de $900^{\circ} \mathrm{C}$, conforme se pode observar. A perda de massa observada para as amostras ao fim do ensaio foi de $42 \%, 61 \%$ e $62 \%$ para as amostras de $5 \%, 10 \%$ e $15 \%$ de CVP, respectivamente.

A perda de massa ocorrida no ensaio para temperaturas superiores a $900^{\circ} \mathrm{C}$ esta associada à redução dos óxidos metálicos, a gaseificação do carbono fixo presente no agente redutor e principalmente devido à volatização de zinco. O zinco após reduzido volatiliza imediatamente, acima de $907^{\circ} \mathrm{C}$. A gaseificação do carbono é bastante desfavorável em temperaturas inferiores, por esta razão que as perdas de massa ocorrem de forma mais rápida entre 900 e $1000^{\circ} \mathrm{C}$.

Verificou-se, conforme a figura 2, que a perda de massa tem uma tendência de ser tanto maior quanto maior o teor de redutor na mistura. A perda de massa da M95/5 é limitada, portanto, pelo suprimento de material carbonoso para o prosseguimento da reação. Desta forma ao ser totalmente consumido o carbono do agente redutor a reação é interrompida. Para a amostra $M_{90 / 10}$ foi verificada uma perda de massa 
próxima àquela máxima teórica (Tabela 6 ) indicando que o suprimento de carbono para esta mistura é suficiente para a redução completa dos óxidos. Já na amostra $M_{85 / 15}$ a perda de massa foi, como para $M_{90 / 10}$, próxima à perda teórica calculada na Tabela 6. Isto mostra que há, nesta condição, excesso de redutor. Sendo, portanto o ponto ótimo de CVP para este pó de aciaria um teor entre 10 e 15\%. Apesar disto é possível observar uma ligeira vantagem cinética no uso da mistura com $15 \%$ de CVP, frente à com $10 \%$.

Os valores teóricos máximos de perda de massa foram apresentados na Tabela 6. Estes valores deveriam ser ligeiramente inferiores aos observados na prática, pois a reação $1 \mathrm{~mol}$ de carbono para $1 \mathrm{~mol}$ de oxigênio redutível considera um excesso de carbono na redução. No entanto os valores observados de perda de massa foram ligeiramente maiores que os calculados na Tabela 6 . Isto possivelmente se deve, como dito anteriormente, a fatores não considerados na estimativa teórica.

\subsection{Testes de Autorredução em Forno Elétrico vertical}

$\mathrm{Na}$ figura 3 são apresentados os resultados dos testes realizados em retorta aquecida por forno elétrico vertical com as pelotas indicadas na tabela 5 .

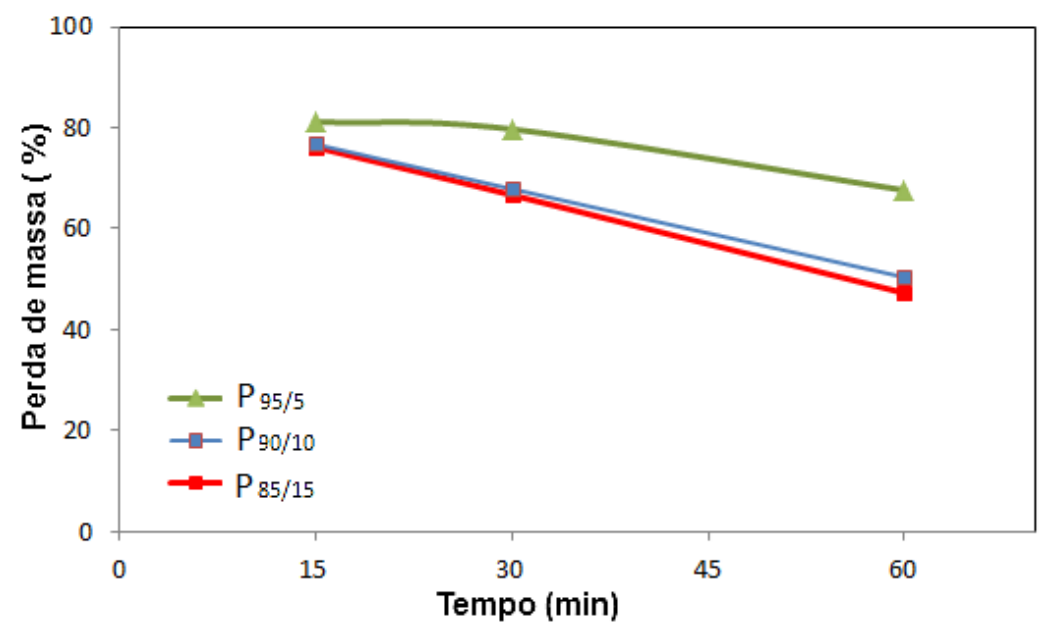

Figura 3. Testes de autorredução em Forno Elétrico Vertical. Onde P PAE/CVP Indica a composição das pelotas.

Pode ser visto na Figura 3 uma perda de massa relativamente pequena (20\%) e próxima para todas as amostras para $15 \mathrm{~min}$ de teste. Estas perdas mássicas aproximadas mesmo para pelotas de diferentes teores de CVP, se deve possivelmente, a um tempo insuficiente de ensaio para o consumo do carbono do redutor contido na pelota de menor de CVP. Desta forma a perda mássica para esta é equivalente às pelotas com maiores teores de redutor. A verificação das perdas de massa ocorrida para 30min de ensaio, no entanto, mostra que $P_{90 / 10}$ e $P_{85 / 15}$ têm perdas mássicas superiores a $P_{95 / 5}$. Com 60min de ensaio é possível observar que as perdas de massa das pelotas foram de 39, 54 e 56\% para as pelotas com 5, 10 e $15 \%$ de CVP, respectivamente.

Neste teste foi possível observar que, assim como ocorreu nos ensaios em termobalança, a perda de massa foi tanto maior quanto maior os teores de redutor nas misturas. As perdas de massa das pelotas com 10 e 15\% de CVP foram próximas, análogo ao que observado em termobalança (figura 2). Estes valores são próximos aos da perda de massa máximo teórico, conforme a tabela 6 . 


\subsection{Análises das fases por Difração de Raios X}

Foram realizadas análises de Difração de raios $X$ nas pelotas após o ensaio de redução em forno elétrico vertical de $1 \mathrm{~h}$. Estas são apresentadas na figura $7 \mathrm{~b}-\mathrm{d}$ e comparadas com a do PAE (Figura 7a).
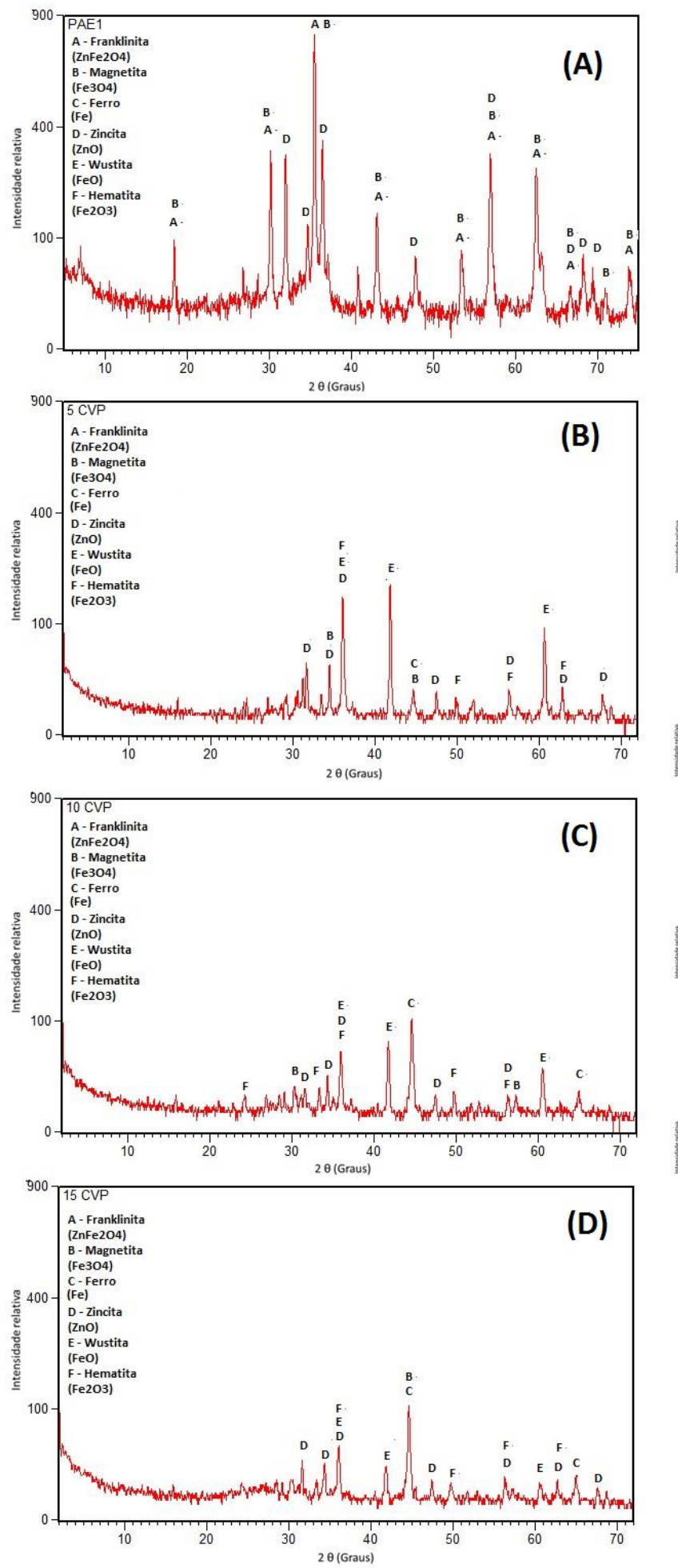

Figura 4 - Testes de difração de raios $x$ - (A) para o PAE (B) Pelota 5\% CVP após $1 \mathrm{~h}$ forno poço (C) Pelota 10\% CVP após $1 \mathrm{~h}$ forno poço (D) Pelota 15\% CVP após $1 \mathrm{~h}$ forno elétrico vertical. 
Conforme mostrado na Tabela 2 as fases que compõem o Pó de aciaria são majoritariamente Franklinita ( $\mathrm{ZnFe2O} 4)$, Magnetita (Fe3O4) e Zincita ( $\mathrm{ZnO})$. Essas são as fases que aparecem de forma mais relevante do difratograma da amostra de PAE (Figura 4a). Após submeter as pelotas a ensaios de $1 \mathrm{~h}$ a $1000^{\circ} \mathrm{C}$ em forno poço, é possível observar que indiferente ao teor de redutor no aglomerado, a fase Franklinita não foi mais observada nas amostras. A amostra com 5\% de redutor apresenta principalmente picos referentes às fases óxidas de ferro (Hematita, Magnetita e Wustita) e de zinco (Zincita), além do surgimento de um pico sobreposto de Ferro metálico e Magnetita. Já para as amostras com teores de redutor de 10 e $15 \%$, as fases óxidas de ferro e zinco também estão presentes, mas se observa 0 surgimento da fase ferro metálico individual, além do anteriormente citado. 0 surgimento de ferro metálico se dá às custas da diminuição de intensidade das fases óxidas.

O completo desaparecimento da fase Franklinita, já na amostra com 5\% de CVP não pode ser explicado por sua completa redução carbotérmica, pois conforme abordado anteriormente, essa amostra possuí carbono abaixo da quantidade necessária para a redução completa das fases óxidas da pelota. Assim acredita-se que com a elevação da temperatura do material ocorra à decomposição da Franklinita em Hematita e Zincita. Essa hipótese foi demostrada em outros trabalhos [12]. Nas pelotas onde acredita-se que o carbono inicial era suficiente para a total redução dos óxidos, os difractogramas mostram que a redução completa não foi possível. Podese relacionar esta ocorrência com a curva de massa obtida para o ensaio em forno poço, Figura 3. Nesta nota-se as curvas de perda de massa das pelotas com 10 e $15 \%$ de CVP não tendem a um patamar. Elas mantem um declividade, indicando que ainda está ocorrendo redução, ou seja, a redução completa dos óxidos ainda não ocorreu.

Paralelo à decomposição térmica da Franklinita a redução dos óxidos sucede. Um indicativo para isto pode ser notado pelos picos em que são identificados apenas a zincita que reduziram de intensidade para com o aumento de teor de redutor nas pelotas. O zinco é reduzido e volatiliza $\left(907^{\circ} \mathrm{C}\right)$ e desta forma ele não aparece em nenhuma análise de DRX como Zn metálico.

Para as pelotas com $10 \%$ de CVP ( $\left.\mathrm{P}_{90 / 10}\right)$ observou-se o aumento dos picos de Ferro metálico em paralelo a redução dos picos de Zincita e fases de ferro de maior oxidação. À medida que estas fases mais oxidadas vão reduzindo de intensidade ou desaparecendo, picos de fases menos oxidadas e de ferro metálico aumentam de intensidade. $O$ que pode ser um indicativo do caminho da reação para a redução progressiva dos óxidos de ferro, como o esperado.

Para as pelotas com 15\% de CVP $\left(\mathrm{P}_{85 / 15}\right)$ observou-se um leve aumento no pico do ferro metálico, assim como diminuição e desaparecimento de picos de óxidos de ferro e zincita.

\section{CONCLUSÕES}

A análise da redução do PAE amostrado foi feita por avaliação da perda de massa e análise de fases via Difração de raios x. Destas análises os seguintes itens são destacados:

- As misturas autorredutoras ensaiadas em termobalança tiveram perdas de $42 \%, 61 \%$ e $62 \%$ para as amostras de $5 \%, 10 \%$ e $15 \%$ de CVP, respectivamente; 
- A perda de massa das pelotas autorredutoras no ensaio de autorredução em forno elétrico vertical foi de 39, 54 e $56 \%$ para as pelotas com 5,10 e $15 \%$ de CVP, respectivamente.

- Tanto as perdas de massa das misturas ensaiadas em termobalança, quanto às pelotas utilizadas em teste de redução em forno elétrico vertical, tiveram para as amostras com 10 e 15\% de CVP perdas de massa próximas entre si e próximas às máximas teóricas. Indicando que o valor ótimo de CVP para um aglomerado com este PAE se situa neste intervalo;

- Através da avaliação das fases por Difração de raios $X$ pode-se observar a decomposição da Franklinita para pelotas ensaiadas em Forno elétrico vertical por $1 \mathrm{~h}$ em $1000^{\circ} \mathrm{C}$.

- Verificou-se a redução da intensidade dos picos nos difratogramas de fases de ferro mais oxidadas à custa do aumento de fases menos oxidadas e aparecimento de Ferro metálico para as pelotas com diferentes teores de CVP. Indicando a redução progressiva dos óxidos de ferro contidos no PAE.

\section{Agradecimentos}

Os autores agradecem ao professor Antônio C. F. Vilela, Coordenador do Laboratório de Siderurgia, pela oportunidade de experiência junto ao laboratório, à Fundação Luiz Englert pelo auxílio financeiro à pesquisa, à Universidade Federal do Rio Grande do Sul, ao LaSid (Laboratório de Siderurgia).

\section{REFERÊNCIAS}

1 Machado JGMS, Estudo da Caracterização e Avaliação da Influência do Tempo na Redução Carbotérmica do Pó de Aciaria Elétrica. (Dissertação de Mestrado) - Porto Alegre,2004.

2 Alves JO, Espinosa DCR, Tenório JAS., Recycling of Steelmaking Slag Aiming at the Production of Thermo Accoustic Insulation, TMS 2009, 138 th Annual Meeting and Exibithion2009, San Francisco, USA.

3 Guézennec AG, HuberJC, Patisson F, Sessiesc P, Birat JP, Ablitzer D, Dust Formation in Electric Arc Furnace: Birth of the Particles, Powder Technology, 2005.

4 Steel Statistical Yearbook 2015. Disponível em http://www.worldsteel.org/dms/internetDocumentList/statistics-

archive/yearbookarchive/Steel-Statistical-Yearbook-2014/document/Steel-StatisticalYearbook-2015.pdf

5 JuergenRuetten; Ron Crittendon. Environmental Friendly recycling of EAF steel milldust - GSD'S state-of-the-art Waelz Process.

6 Adolfo P. Zambrano; Cyro Takano; Marcelo B. Mourão; Solon Y. Tagusagawa. Influence of the Binder on the Mechanical Properties of the Chromite Self-Reducing Pellets. IJBHT.2013

7 Felipe Ferreira Buboltz, Bruno Deves Flores, Pedro Jorge WalburgaKeglevich de Buzin, Nestor Cezar Heck, Eduardo Osório, Antônio Cezar Faria Vilela - Caracterização e avaliação da redução carbotérmica via termogravimetria de Pó de Aciaria Elétrica, Porto Alegre - 2015.

8 Chang, H. S.: Taiwan 1999 Educational ProgrammeonWasteTreatmentof EAF SlagandDust, Taichung, Taiwan, 19-21 de outubro de 1999, Taiwan Steel \& Iron Industries Association, Taipei, Taiwan, 1999.

9 Anderson Azevedo Agra, Helioir Antonio da Silva Júnior, Matheus Frederico Rückert, Ligia dos Santos de Oliveira, Felipe Buboltz Ferreira, Bruno Deves Flores, Antônio Cezar Faria Vilela- Avaliação da utilização de diferentes aglomerantes nas 
propriedades mecânicas de pelotas autorredutoras de pó de aciaria, Porto Alegre 2015.

10 Buzin, P. J. W. K., Desenvolvimento de briquetes autorredutores a partir de carepas de processamento siderúrgico para utilização em forno elétrico a arco (Dissertação de mestrado), Porto Alegre, 2009.

11 Bagatini, M. C., Estudo da reciclagem de carepa através de briquetes autorredutores para uso em forno elétrico a arco (Tese de doutorado), Porto Alegre, 2001.

12 Jyh-Jen Lee, Chun-I Lin, Hsi-Kuei Chen - Carbothermal Reduction of Zinc Ferrite Metallurgical and Materials Transactions - Taiwan, 2000. 